\title{
EVALUACIÓN DE TÉCNICAS ANESTÉSICAS GOWGATES Y AKINOSI EN NERVIO DENTARIO INFERIOR - RAMIFICACIONES, EN CLÍNICA ODONTOLÓGICA, UCSG, 2015 EVALUATION OF GOWGATES AND AKINOSI ANESTHETIC TECHNIQUES IN INFERIOR DENTAL NERVE-RAMFICATIONS, IN A DENTAL CLINIC, UCSG, 2015 AVALLÇ̄̃̄O DAS TÉCNICAS ANESTÉSICAS GOWGATEE AKINOSI NAS RAMIFICAÇÕES DO NERVO DENTÁRIO INFERIOR, EM CLIIIICA ODONTOLÓGICA, UCSG, 2015
}

\author{
JUAN CARRERA MOSQUERA', CARLOS YCAZA REYNOSO'
}

${ }^{1}$ Universidad Católica de Santiago de Guayaquil; Guayaquil, Ecuador

RESUMEN

Introducción: existen diferentes técnicas anestésicas para lograr la inhibición sensorial del nervio dentario inferior, ramificaciones y estructuras. Cada una tiene diferentes resultados que muestran eficacia en casos distintos. Objetivo: revisar clínicamente las técnicas de anestesia para establecer la eficacia de ambas y el dolor que causa cada una con respecto a la otra. Diseño: se realizó un estudio clínico de tipo analítico correlacional de corte transversal, evaluando 100 historias clínicas de pacientes mayores de edad que fueron atendidos en la clínica odontológica - UCSG. Resultado: el estudio reveló la eficacia de ambas técnicas anestésicas dentro del $50 \%$ de la técnica GowGates y el $50 \%$ de la técnica Akinosi. De acuerdo a las variables establecidas, ambas técnicas presentan diferentes resultados pero no existe una diferencia pronunciada. Conclusión: en el $100 \%$ de los pacientes se evidencia que el dolor a la punción es similar en ambas técnicas, de acuerdo con el periodo de latencia se concluye que los resultados varían en pequeñas proporciones y la inhibición del dolor en ambas es eficaz con pequeñas diferencias. En conclusión se establece que ambas técnicas son eficaces.

PALABRAS CLaVE: tiempo de reacción, eficacia, anestesia, dolor, puntos anatómicos de referencia, técnicas, terminaciones nerviosas.

ABSTRACT

Introduction: there are different anesthetic techniques to achieve sensory inhibition of the lower dental nerve, branches, and structures. Each one has different results that show efficacy in different cases. Objective: clinically review anesthesia techniques to establish the efficacy of both and the pain caused by each one concerning the other. Design: a cross-sectional correlational analytical-type clinical study was conducted, evaluating 100 medical records of patients of legal age who were treated at the Dental Clinic - UCSG. Result: the study revealed the efficacy of both anesthetic techniques within $50 \%$ of the GowGates technique and $50 \%$ of the Akinosi technique. According to the established variables, both techniques present different results but there is no pronounced difference, discrepancies are small. Conclusion: in $100 \%$ of the patients, is evidenced that the pain at puncture is similar in both techniques, according to the latency period it was concluded that the results vary in small proportions and, the inhibition of pain in both is effective with small discrepancies. In this sense, it is was established that both techniques are effective.

KEYWORDS: reaction time, efficacy, anesthesia, causalgia, anatomical landmarks, techniques, nerve endings.

RESUMO

Introdução: existem diferentes técnicas anestésicas para atingir a inibição sensorial do nervo, ramos e estruturas dentais inferiores. Cada um tem resultados diferentes que mostram eficácia em casos diferentes. Objetivo: revisar clinicamente as técnicas de anestesia para estabelecer a eficácia de ambas e a dor causada por cada uma em relação à outra. Delineamento: foi realizado um estudo clínico transversal, correlacional, do tipo analítico, avaliando 100 prontuários de pacientes idosos atendidos na Clínica Odontológica - UCSG. Resultado: 0 estudo revelou a eficácia de ambas as técnicas anestésicas em $50 \%$ da técnica de GowGates e $50 \%$ da técnica de Akinosi. De acordo com as variáveis estabelecidas, as duas técnicas apresentam resultados diferentes, mas não há diferença pronunciada, as diferenças são pequenas. Conclusão: em $100 \%$ dos pacientes evidencia-se que a dor à punção é semelhante nas duas técnicas, de acordo com o período de latência conclui-se que os resultados variam em pequenas proporções e a inibição da dor em ambas é efetiva com pequenas discrepâncias. Nesse sentido, fica estabelecido que ambas as técnicas são eficazes. Palavras-chave: tempo de reação, eficácia, anestesia, dor, marcos anatômicos, técnicas, terminações nervosas.

PALAVRAS-CHAVE: tempo de reação, eficacia, anestesia, causalgia, marcos anatómicos, técnicas, terminações nervosas. 
INTRODUCCIÓN

En la variedad de técnicas anestésicas para extracción de órganos dentales o diferentes procedimientos quirúrgicos, usamos infiltrativas para inhibir nervios localizadamente, dos técnicas: Akinosi y Gow Gates. El objetivo de ambas es inhibir localmente el nervio mandibular que se refleja en el nervio mentoniano por encontrarse en el trayecto anterior. ${ }^{24,26}$

La técnica de Gow gates creada por el Doctor Australiano George Gow Gates en el año 1973 12,16, es de tipo local, considerada poco compleja, es utilizada para procedimientos de la cavidad oral, su objetivo es inhibir sensorialmente el nervio dentario inferior que abarca un hemisferio de la mandíbula, compromete las ramas intraorales que son mentoniana, incisiva, lingual, milohioidea, auriculotemporal, bucal e inhibe corticales internas, externas, órganos dentales, y a su vez la mucosa, piso de la boca y dos tercios anteriores de la lengua. $1,2,4-6,17-23,25,27$,

La técnica Vazirani-Akinosi fue creada por el Doctor Vazirani en el año 1960, fue llamada técnica de bloqueo mandibular con boca cerrada ${ }^{12,16}$, y en 1977.

Akinosi la describió. El objetivo es inhibir el nervio dentario inferior a su vez los órganos dentales y las ramificaciones de este nervio citadas anteriormente. Es conocida como la técnica de boca cerrada, se utiliza en pacientes con trismus, que no tienen una apertura bucal extensa o en pacientes que presenten inseguridad al momento de una intervenció. - $^{6-11,13-15,28,30}$ Con estos antecedentes, es oportuno determinar la eficacia de la técnica CowGates y Akinosi, la susceptibilidad al dolor frente a estas técnicas.

\section{METODOLOGÍA}

El presente estudio clínico es de tipo analítico correlacional de corte transversal, basado en las recomendaciones de la declaración de Helsinki. contó con un universo constituido por cada paciente que presentaba la historia clínica adecuada con los datos previos para poder proceder a realizar el estudio. Todos los pacientes seleccionados fueron mayores de edad, se les realizo preguntas previas sobre el estado de salud general del paciente y sus antecedentes médicos, se tomó como criterio de exclusión a los pacientes que presenten enfermedades sistémicas, mayores de 60 años de edad, pacientes que se opongan a la publicación de su información y pacientes que presenten una deficiencia mental. Después se procedió a evaluar a los pacientes durante el momento de la infiltración de la anestesia y posterior a esto también, esta evaluación se realizó a los 100 pacientes y se basó en preguntas de acuerdo a las variables planteadas. El studio fue realizado en la Clínica Odontológica de la Universidad Católica Santiago de Guayaquil-durante el segundo semestre del año 2015.

Toda la información fue tabulada y documentada, seguida por las pruebas estadísticas. La validación de las estadísticas se realizó mediante la prueba chi cuadrado, con grado de validación de 0,05 para $95 \%$ de contabilidad si $\mathrm{p} \leq 0,05$. Se realizó historia clínica a cada paciente con las respectivas preguntas con el fin de recopilar información para realizar el estudio.

RESULTADOS

En el gráfico 1, se realizó estudio de un Universo de 100 pacientes, se realizaron 50 muestras de técnicas de anesthesia de tipo Gow Gates y 50 muestras de anesthesia de tipo Akinosi.

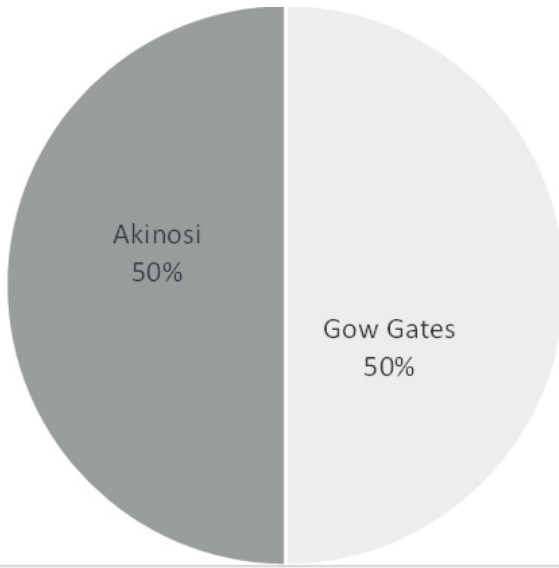

Figura 1. Distribución porcentual de las muestras clínicas obtenidas, 50 pacientes anestesiados con técnica Gow Gates y 50 pacientes anestesiados con técnica Akinosi.

Fuente: paciente de clínica Odontológica de la Universidad Católica Santiago de Guayaquil.

El análisis estadístico bivariable con respecto al dolor de la punción e infiltración del anestésico reveló los siguientes resultados: el 30\% de la técnica Gow Gates refirió nivel 0 y el 29\% de la técnica Akinosi refirió nivel 0; el 14\% de la técnica GG refirió nivel 1 y el 6\% de la técnica Akinosi refirió 1 ; el 4\% de la técnica GG refirió dolor nivel 2 y el $9 \%$ de la técnica Akinosi refirió nivel 2; el 1\% de la técnica GG refirió dolor nivel 3 y el 4\% de la técnica Akinosi refirió dolor 3; el 1\% de la técnica CG refirió nivel 4 y el 2\% de la técnica Akinosi refirió nivel 4. 


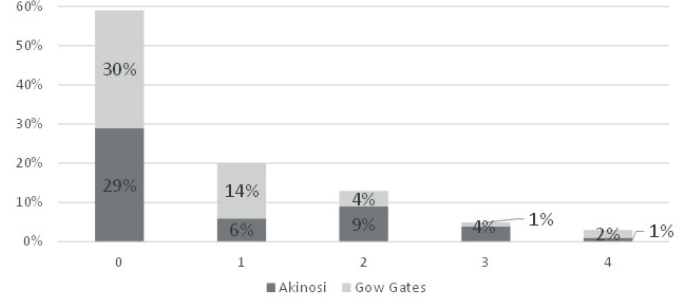

Figura 2. Distribución porcentual de muestras del dolor a la punción en pacientes anestesiados con técnica Gow Gates y Akinosi.

Fuente: paciente de clínica Odontológica de la Universidad Católica de Satiago de Guayaquil.

El resultado obtenido frente al periodo de latencia de la anestesia en el labio es el siguiente: el $42 \%$ de la técnica GG refirió adormecimiento a los 3 minutos y el 33\% de la técnica Akinosi refirió a los 3 minutos; el 7\% de la técnica GG refirió adormecimiento a los 5 minutos y el $10 \%$ de la técnica Akinosi refirió adormecimiento a los 5 minutos; el 1\% de la técnica GG refirió adormecimiento a los 7 minutos y el 5\% de la técnica Akinosi refirió adormecimiento a los 7 minutos.

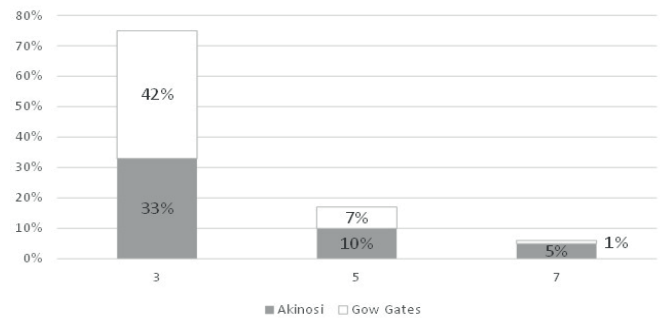

Figura 3. Distribución porcentual de muestras de técnicas de anestesia Gow Gates y Akinosi relacionado entre si, para medir el periodo de latencia sobre labio inferior del hemisferio anestesiado.

Fuente: Paciente de clínica Odontológica de la Universidad Católica Satiago de Guayaquil.

El resultado obtenido frente al periodo de latencia de la anestesia en la lengua es el siguiente: el $42 \%$ de la técnica GG refirió adormecimiento a los 3 minutos y el 33\% de la técnica Akinosi refirió a los 3 minutos; el 6\% de la técnica GG refirió adormecimiento a los 5 minutos y el 13\% de la técnica Akinosi refirió adormecimiento a los 5 minutos; el $2 \%$ de la técnica GG refirió adormecimiento a los 7 minutos y el 4\% de la técnica Akinosi refirió adormecimiento a los 7 minutos.

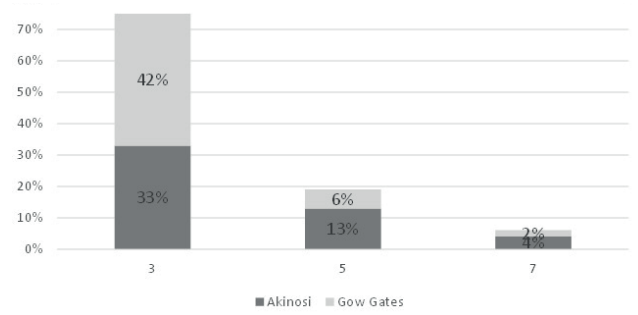

Figura 4. Distribución porcentual de muestras de técnicas de anestesia GowGates y Akinosi relacionadas entre si, para medir el periodo de latencia sobre la hemisferio de la lengua del lado anestesiado.

Fuente: Paciente de clínica Odontológica de la Universidad Católica Satiago de Guayaquil.
El resultado estadístico sobre el adormecimiento vestibular a los 10 minutos luego de poner la anestesia nos da los siguientes datos: el 33\% de la técnica GG y el 35\% de la técnica Akinosi refirieron nivel 0 según la escala de Mc Gill; el $8 \%$ de la técnica GG y el $4 \%$ de la técnica Akinosi refirieron dolor nivel 1; el $9 \%$ de la técnica GG y la técnica Akinosi refirieron nivel 2; el 1\% de la técnica Akinosi refirió dolor 3 y 4.

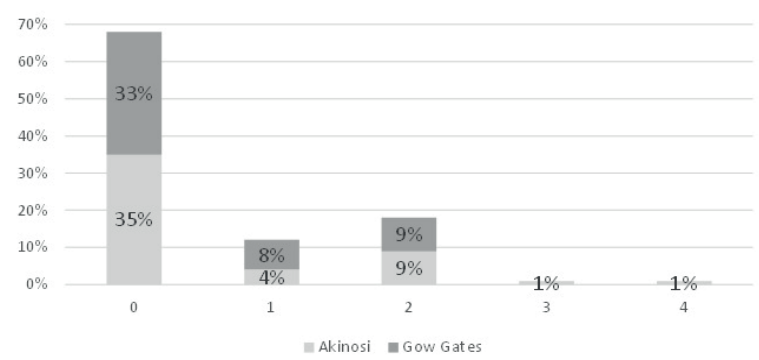

Figura 5. Distribución porcentual de muestras de técnicas de anestesia Gow Gates y Akinosi, midiendo la inhibición sensitiva de la zona vestibular, 10 minutos posterior a la infiltración.

Fuente: paciente de clínica Odontológica de la Universidad Católica Satiago de Guayaquil

El resultado estadístico con respecto al adormecimiento lingual a los 10 minutos de colocada la anestesia arrojo los siguientes resultados: el 41\% de la técnica GG refirió nivel 0 en la escala de Mc Gill y el 34\% de la técnica Akinosi refirió nivel 0; el 9\% de la técnca GG refirió nivel 1 y el $8 \%$ de la técnica Akinosi refirió nivel 1; el 6\% de la técnica Akinosi refirió nivel 2; el 2\% de la técnica Akinosi refirió el nivel 3.

Distribución porcentual de muestras de técnicas Gow Gates y Akinosi, midieron la inhibición sensitive de la zona lingual, 10 minutos posterior a la infiltración.

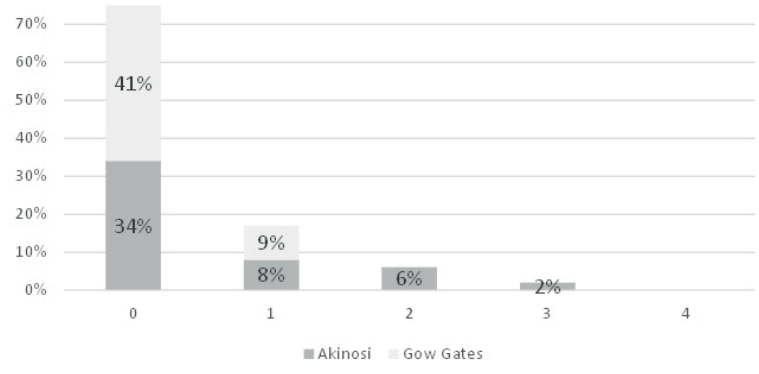

Figura 6. Distribución porcentual de muestras de técnicas de anestesia Gow Gates y Akinosi, midiendo la inhibición sensitiva de la zona lingual, 10 minutos posterior a la infiltración.

Fuente: paciente de clínica Odontológica de la Universidad Católica Satiago de Guayaquil

\section{DISCUSIÓN}

Del 100\% de los pacientes se tomó muestras el 50\% para la técnica Gow Gates y 50\% para Akinosi. Con respecto a la variable del dolor, ambas tuvieron resultados similares con un pequeño margen 
de diferencia. Fue percebido con respecto a los periodos de latencia, que la técnica Gow Gates tuvo ventaja sobre la técnica Akinosi debido a que el efecto de inhibición sensorial era más rápido que la técnica del lado opuesto, pero ambas coincidieron en tener su periodo de latencia más eficaz a los 3 minutos. Y con respecto a la inhibición de estímulos en la zona vestibular tuvo más éxito la técnica Akinosi sobre la GG. Pero en la zona lingual fue lo contrario, la técnica GC dió resultados mas rápidos que la técnica Akinosi.

\section{CONCLUSIÓN}

Fue posible obtener resultado del presente estudio, y podemos concluir con la idea de que ambas técnicas de anestesia son eficaces, con respecto a la variable del dolor; se concluye que ambas técnicas anestésicas tienen su nivel de dolor similar, ya que la diferencia porcentual fue insignificante. De acuerdo con la inhibición sensitiva de la zona anestesiada los autores constataron que ambas técnicas son eficaces igualmente, se acota también que ciertos parámetros tambien influyen en el momento de la inhibición, se toma en cuenta tambien la habilidad del operador y la cantidad de cartuchos que se utilizan en el paciente. Tambien se pudo evidenciar la necesidad de la técnica de akinosi en pacientes que tienen una limitada apertura bucal producto de alguna patología o trauma psicológico. Ambas técnicas prevalecen en la eficacia al momento de realizar algún acto quirúrgico.

\section{REFERENCIAS BIBLIOGRÁFICAS}

1. Bernhard K, Castellon L, German L. GOW GATES Technique: A pilot study for extraction procedures with clinical evaluation and review. American Dental Society of Anesthesiology. (2008); 55:2

2. Fehrenbach M. GOW - GATES mandibular nerve block: an alternative in local anesthetic use. Clinical feature. American Dental Society of Anesthesiology. American Society for the Advancement of Anesthesia in Dentistry. (2002)

3. Reza Jamalpour M, Talimkhani I. Efficacy of the GOW - GATES and inferior alveolar nerve block techniques in providing anesthesia during surgical removal of impacted lower third molar: a controlled randomized clinical trial. Avicena J Dent Res. . (2013) 5(1)

4. Beiner C, Argandoña J, Diaz V, Diaz C. Estudio comparativo entre las tecnicas anestesicas Spix y GOW -ATES. Revista Dental de Chile. (2010)101(3)
5. Steven J. Painless Dentistry. Dentaltown Kisco. (2010)

6. Choque C, Condori E, OrtizS. (2012). Tecnicas de anestesia troncular del maxilar inferior. Revista de Actualización Clínica. Vol. 27 (1329-1333)

7. Haas D. alternative mandibular nerve block techniques. a review of the GOW - GATES AND AKINOSI- VAZIRANI CLOSED - mouth mandibular nerve block techniques. American Dental Association, JADA (2011) 142(9)

8. Goel M, Nagpal R, Bhandari P. efficacy of anesthesia of long buccal nerve in akinosi closed mouth technique - a prospective study. Indian Journal of Dental Sciences. (2011) Vol 5 (2)

9. Verma N, Jeevan L. comparisson and clinical efficacy of mandibular nerve anesthesia by direct conventional techniquewith VAZURANI - AKINOSI mandibular nerve block technique. J. Anaesth Clin Pharmacol (2011) 26(1): $79-82$

10. Mishra S, Tripathy R, Sabhlok S, Kaumar Panda P, Patnaik S. comparative analysis between direct conventional mandibular nerve block and VAZIRANI-AKINOSI closed mouth mandibular nerve block technique. International Journal of Advancements in Researchs and Technology. (2012) Vol. 1 (6)

11. Martinez-G J,Peña B, Fernandez C,Hipolito MArin L.S, Peñarrocha D. estudio comparativo entre el loqueo mandibular directo y la tecnica de AKINOSI. Medicina Oral S.L. (2003) Vol. 8 (143-9)

12. Martinez A. Anestesia Bucal Guia Practica. Editorial Panamericana. (2009) 978-958-844303-4 ISBN.

13. González S. Héctor, Peñarrocha D. Miguel, Guarinos C. Juan, Balaguer M. José. Pain and inflammation in 41 patients following the placement of 131 dental implants. (2005) Med Oral Patol Oral Cir Bucal.

14. Stubblefield. Michael D, Manfield. Laura, Riedel. Elyn R. A preliminary report on the efficacy of a dynamic jaw opening device as part of the multimodal treatment of trismus in patient $s$ with head and neck cancer. ARCH (2010) Phys Med Rehabil. Vol 91

15. Oltra. E, Gonzalez. C, Mendiolagoitia. L, Sanchez. P. suturas y cirugia menor. 2nda Ed. Buenos Aires. (2007) Editorial Panamericana.

16. Martinez. Adel. Anestesia bucal guia practica. (2009) Editorial Medica Panamericana. Pag. 2.

17. Segura Mungia. S. diccionario etimologico de medicina. Universidad de Deusto. (2014) Pag. 37. ISBN: 978-84-15772-88-0. 
18. Miller. Ronald D, Eriksson. L, Fleisher. L, Young. William. Anestesia. Septima Edicion. Elsevier. (2010) Vol. 1.

19. Leon. M. Anestésicos locales en odontología. Corporación editora Medica del Valle. Revista Colombia Medica. (2001) Vol. 23. Pg 137 - 140. ISSN: 1657.9534.

20. American Dental Association. Terapeutica dental. Masson.Edición Española. (2003) Pag. 2.

21. J. Serra Catafu. Tratado de dolor neuropático. Editorial Medica Panamericana. (2006) Pag. 17

22. Pendola. T. Anestesicos locales su uso en odontologia. 1era ed. Talleres Direccion de Docencia Facultad Odontologia Departamento de estomatología quirúrgica Universidad de Concepcion. (2007)

23. Ferrandiz Mach M.Fiosiopatologia del dolor. Hospital de la Santa Creu i Sant Pau; (2007) 1-14.

24. Malamed S. MANUAL DE ANESTESIA LOCAL. (2006) $5^{\underline{a}}$ ed. Madrid: Elsevier Mosby;

25. M. Latarjet, A. Ruiz Liard. Anatomia humana. (1995) 3era ed. Vol. 1.España. Editorial Panamericana. Pag. 340-347.
26. Macouzet Olivar. C. Anestesia local en odontologia. (2008) 2nda ed. Mexico. Editorial El Manual Moderno. Pag 120.

27. Garcia-Peñin. A, Guisado. B, Montalvo. Juan J. riesgos y complicaciones de anestesia local en la consulta dental. Estado actual. RCOE. Madrid. (2003) Vol. 8.

28. Martinez. J, Peña. B, Fernandez. F, Hipolito. L, Peñarrocha. D. Estudio comparativo entre el bloqueo mandibular directo y la tecnica AKINOSI. Medicina oral. (2003) Vol. 8: 143-9.

29. Almeida. F, Almeida. M, Groppo. F. Comparison of pain intensity during inferior alveolar nerve block. Rev Dor. Sao Paulo (2013) 14(3): 165-8.

30. Lenka. S, Nikil. J, Mohanty. R, Singh. D, Minkle. G. A Clinical comparison of three techniques of mandibular local anaestheSIA. Journal of Research and Advancement in Dentistry. (2013) 2:3:61: -67 